\title{
Light Propagation Effects In QED: Effective Action Approach
}

\author{
Y. M. Chd * \\ Department of Physics, College of Natural Sciences, \\ Seoul National University, Seoul 151-742, Korea \\ D. G. Pali \\ Center for Theor. Physics, Seoul National University, Seoul 151-742, Korea and \\ Institute of Applied Physics, Uzbekistan National University, Tashkent 700-095, Uzbekistan \\ M. L. Walket \\ Department of Physics, Chiba University, 1-33 Yayoi-Cho, Inage-ku, Chiba 263-8522, Japan

\begin{abstract}
The effects of light propagation in constant magnetic and electric backgrounds are considered in the framework of the effective action approach. We use the exact analytic series representation for the one-loop effective action of QED and apply it to the birefringence effect. Analytical results for the light velocity modes are obtained for weak and strong field regime. We present asymptotic formulae for the light velocity modes for the ultra strong magnetic field which can be realized in some neutron stars.
\end{abstract} \\ PACS numbers: 12.20.-m, 41.20.Jb, 11.10.Ef \\ Keywords: quantum electrodynamics, effective action, birefringence
}

\section{INTRODUCTION}

It is well known that the Euler-Heisenberg effective Lagrangian in quantum electrodynamics (QED) is presented by an asymptotic series [1]. Since the asymptotic series is divergent its applications are limited to a weak field approximation. For the problems related to a strong field background one has to apply the complete expressions for the effective action. The effective action at one- and two-loop level for special electromagnetic backgrounds have been studied in [2, 3] (see as well [4] and refs. therein). Recently the analytic series representation for the one-loop effective action of QED has been constructed [5] from Schwinger's integral expression for the effective action [6]. The knowledge of an explicit analytical expression of the effective action is useful for studying quantum effects like photon splitting [7] and photon propagation in electric and magnetic fields [8, 9, 10, 11]. Another motivation comes from the theoretically predicted existence of strong magnetic fields of magnitude $B$ above the critical value $B_{c r}=\frac{m^{2}}{e} \simeq 5 \cdot 10^{13} G$ in the cores of some neutron stars [12].

In the present paper we consider light propagation effects in the constant magnetic and electric field in the framework of the effective action approach proposed in 11] applying the series representation for the one-loop QED effective action [5]. In Section [1] we present some general formulae relevant to the effective action approach

\footnotetext{
*Electronic address: ymcho@yongmin.snu.ac.kr

${ }^{\dagger}$ Electronic address: dmipak@phya.snu.ac.kr

${ }^{\ddagger}$ Electronic address: m.walker@aip.org.au
}

11. In sections III and IV we consider photon propagation in the pure magnetic and pure electric external field correspondingly. Birefringence effect in the magnetic and electric field is considered in sections $\nabla \mathbf{V I}$ Some technical details of calculation and useful formulae are collected in the appendix.

\section{LIGHT CONE CONDITION IN THE EFFECTIVE ACTION FORMALISM}

The integral representation for the one-loop effective action of QED for slowly varying background fields has been known for a long time [ []

$$
\Delta \mathcal{L}=-\frac{e^{2}}{8 \pi^{2}} a b \int_{0}^{\infty} \frac{d t}{t} \operatorname{coth}(e a t) \cot (e b t) e^{-m^{2} t},
$$

where $a, b$ are gauge invariant variables corresponding to magnetic and electric fields in a Lorentz frame where the both field vectors are parallel

$$
\begin{aligned}
& a=\frac{1}{2} \sqrt{\sqrt{F^{4}+(F \tilde{F})^{2}}+F^{2}}, \\
& b=\frac{1}{2} \sqrt{\sqrt{F^{4}+(F \tilde{F})^{2}}-F^{2}} \\
& F^{2}=F_{\mu \nu} F^{\mu \nu} \\
& \tilde{F}^{\mu \nu}=\frac{1}{2} \epsilon^{\mu \nu \rho \sigma} F_{\rho \sigma} .
\end{aligned}
$$

We start from the series representation for the oneloop effective Lagrangian [5] which is more suitable in physical problems when we need explicit analytical ex- 
pression of the effective Lagrangian

$$
\begin{aligned}
& \mathcal{L}=-\frac{a^{2}-b^{2}}{2} \\
& -\frac{e^{2}}{4 \pi^{3}} a b \sum_{n=1}^{\infty} \frac{1}{n}\left[\operatorname { c o t h } ( \frac { n \pi b } { a } ) \left(\operatorname{ci}\left(\frac{n \pi m^{2}}{e a}\right) \cos \left(\frac{n \pi m^{2}}{e a}\right)\right.\right. \\
& \left.+\operatorname{si}\left(\frac{n \pi m^{2}}{e a}\right) \sin \left(\frac{n \pi m^{2}}{e a}\right)\right)-\frac{1}{2} \operatorname{coth}\left(\frac{n \pi a}{b}\right)\left(\exp \left(\frac{n \pi m^{2}}{e b}\right)\right. \\
& \left.\left.\cdot \operatorname{Ei}\left(-\frac{n \pi m^{2}}{e b}\right)+\exp \left(-\frac{n \pi m^{2}}{e b}\right) \operatorname{Ei}\left(\frac{n \pi m^{2}}{e b}\right)\right)\right] \\
& \quad \equiv \mathcal{L}_{c l}+\delta \mathcal{L}
\end{aligned}
$$

where $\mathcal{L}_{c l}$ is the classical QED Lagrangian.

We will follow the effective action approach [11] to study the light propagation effects in non-trivial vacua. The light cone condition for a soft photon propagating in the homogeneous electromagnetic background (neglecting the back-reaction effects) can be derived from the equation of motion corresponding to the full effective action. The final expression for the light cone condition is the following [1]

$$
\begin{aligned}
0 & =\left(\partial_{x} \mathcal{L}\right) k^{2}+M_{\alpha \beta}^{\mu \nu} k_{\mu} k^{\alpha} \epsilon^{\beta} \epsilon_{\nu}, \\
\bar{k}^{\mu} & =\frac{k^{\mu}}{|\mathbf{k}|} \equiv(v, \hat{\mathbf{k}}),
\end{aligned}
$$

where $\epsilon^{\mu}$ is a unit polarization vector of the photon, and $M_{\alpha \beta}^{\mu \nu}$ is defined by (in the Lorenz gauge $k_{\mu} \epsilon^{\mu}=0$ )

$$
\begin{aligned}
M_{\alpha \beta}^{\mu \nu} \equiv & F^{\mu \nu} F_{\alpha \beta}\left(\partial_{x}^{2} \mathcal{L}\right)+\tilde{F}^{\mu \nu} \tilde{F}_{\alpha \beta}\left(\partial_{y}^{2} \mathcal{L}\right) \\
& +\partial_{x y} \mathcal{L}\left(F^{\mu \nu} \tilde{F}_{\alpha \beta}+\tilde{F}^{\mu \nu} F_{\alpha \beta}\right), \\
x \equiv & \frac{1}{4} F_{\mu \nu} F^{\mu \nu}=\frac{1}{2}\left(a^{2}-b^{2}\right), \\
y \equiv & \frac{1}{4} F_{\mu \nu} \tilde{F}^{\mu \nu}=a b .
\end{aligned}
$$

The matrix $M_{\alpha \beta}^{\mu \nu}$ provides the quantum corrections to the classical light cone condition $k^{2}=0$. The light cone condition can be also transformed into another form which is more suitable for calculation of the light velocity averaged over polarizations. After averaging over the polarization states of the photon the light cone condition can be rewritten as 11]

$$
\bar{v}^{2}=1-Q<T^{\mu \nu}>\bar{k}_{\mu} \bar{k}_{\nu},
$$

here, $\left\langle T^{\mu \nu}\right\rangle$ is a vacuum expectation value (VEV) of the electromagnetic energy-momentum tensor, and $Q$ is an effective action charge defined in terms of the effective Lagrangian and its derivatives [11]. For convenience we give the definition of the effective action charge $Q$ in terms of gauge invariant variables $a, b$

$$
\begin{gathered}
Q=\left(\partial_{a}^{2} \mathcal{L}+\partial_{b}^{2} \mathcal{L}\right)\left(\mathcal{L}\left(\partial_{a}^{2} \mathcal{L}+\partial_{b}^{2} \mathcal{L}\right)+\right. \\
\left.\frac{1}{a^{2}+b^{2}}\left(a \partial_{a} \mathcal{L}-b \partial_{b} \mathcal{L}\right)^{2}-a \partial_{a} \mathcal{L} \partial_{b}^{2} \mathcal{L}-b \partial_{b} \mathcal{L} \partial_{a}^{2} \mathcal{L}\right)^{-1}
\end{gathered}
$$

As can be seen from the light cone condition, Eq. (6), the light velocity is determined by the product of the effective action charge $Q$ and the VEV of the energymomentum tensor $\left\langle T_{\mu \nu}\right\rangle$. It is convenient to introduce the following notations for the combinations of special functions

$$
\begin{aligned}
& g(x)=\operatorname{ci}(x) \cos x+\operatorname{si}(x) \sin x, \\
& h(x)=\frac{1}{2}\left[e^{x} \operatorname{Ei}(-x)+e^{-x} \operatorname{Ei}(x)\right] .
\end{aligned}
$$

The properties of the special functions are given in 13 , 14]. Using Eq. (3) for the one-loop correction $\delta \mathcal{L}$, one can write down explicit expressions for the derivative terms

$$
\begin{aligned}
\partial_{a} \delta \mathcal{L} & =\frac{1}{a} \delta \mathcal{L}-\frac{e^{2}}{4 \pi^{3}} \sum_{n=1}^{\infty}\left[\frac{\pi b^{2}}{a} \operatorname{csch}^{2} \beta g(z)\right. \\
+ & \left.\pi a \operatorname{csch}^{2} \alpha h(\bar{z})-\frac{\pi m^{2} b}{e a} g^{\prime}(z) \operatorname{coth} \beta\right], \\
\partial_{b} \delta \mathcal{L} & =-\frac{e^{2}}{4 \pi^{3}} \sum_{n=1}^{\infty}\left[\left(\frac{a}{n} \operatorname{coth} \beta-\pi b \operatorname{csch}^{2} \beta\right) g(z)\right. \\
- & \left(\frac{a}{n} \operatorname{coth} \alpha+\frac{\pi a^{2}}{b} \operatorname{csch}^{2} \alpha\right) h(\bar{z}) \\
+\quad & \left.\frac{\pi m^{2} a}{e b} h^{\prime}(\bar{z}) \operatorname{coth} \alpha\right], \\
\partial_{a}^{2} \delta \mathcal{L} & =\frac{e^{2} b}{4 \pi^{3}} \sum_{n=1}^{\infty}\left[\frac{1}{n a} \operatorname{coth} \beta+g(z) \operatorname{coth} \beta\left(\frac{n \pi^{2} m^{4}}{e^{2} a^{3}}\right.\right. \\
- & \left.\frac{2 b^{2} n \pi^{2}}{a^{3}} \operatorname{csch}^{2} \beta\right)+\frac{2 b n \pi^{2} m^{2}}{e a^{3}} \operatorname{csch}^{2} \beta g^{\prime}(z) \\
+\quad & \left.\left(\frac{2 a n \pi^{2}}{b^{2}} \operatorname{coth}^{2} \alpha-\frac{2 \pi}{b}\right) \operatorname{csch}^{2} \alpha h(\bar{z})\right], \\
\partial_{b}^{2} \delta \mathcal{L} & =\frac{e^{2} a}{4 \pi^{3}} \sum_{n=1}^{\infty}\left[-\frac{1}{b n} \operatorname{coth} \alpha+h(\bar{z}) \operatorname{coth}^{2}\left(\frac{n \pi^{2} m^{4}}{e^{2} b^{3}}\right.\right. \\
+\quad & \left.\frac{2 a^{2} n \pi^{2}}{b^{3}} \operatorname{csch}^{2} \alpha\right)-\frac{2 a n \pi^{2} m^{2}}{b^{3} e} \operatorname{csch}^{2} \alpha h^{\prime}(\bar{z}) \\
+\quad & \left.\left(\frac{2 \pi}{a}-\frac{2 b \pi^{2} n}{a^{2}} \operatorname{coth} \beta\right) \operatorname{csch}^{2} \beta g(z)\right],
\end{aligned}
$$

where

$$
\begin{aligned}
z=\frac{\pi n m^{2}}{e a}, & \bar{z}=\frac{\pi n m^{2}}{e b}, \\
\alpha=\frac{\pi n a}{b}, & \beta=\frac{\pi n b}{a} .
\end{aligned}
$$

The vacuum averaged value of the energy-momentum tensor can be found from the effective Lagrangian as in [1]

$$
\begin{aligned}
<T^{\mu \nu}>=- & \frac{T_{c l}^{\mu \nu}}{a^{2}+b^{2}}\left(a \partial_{a} \mathcal{L}-b \partial_{b} \mathcal{L}\right)+ \\
& g^{\mu \nu}\left[\mathcal{L}-\frac{1}{2}\left(a \partial_{a} \mathcal{L}+b \partial_{b} \mathcal{L}\right)\right],
\end{aligned}
$$

here, $T_{c l}^{\mu \nu}$ is the classical energy-momentum tensor

$$
T_{c l \nu}^{\mu}=F^{\mu \rho} F_{\nu \rho}-\frac{a^{2}-b^{2}}{2} \delta_{\nu}^{\mu} .
$$


In the following we will apply the above general equations to special cases of light propagation in homogeneous pure magnetic and electric background.

\section{LIGHT VELOCITY IN MAGNETIC FIELD}

Light propagation in the presence of a constant magnetic field in one-loop approximation was first studied in 7, 8, 9] and later by many others (see refs. in [4]). The light velocity in the weak magnetic field was well studied using the Euler-Heisenberg effective Lagrangian [1] (weak-field limit of the complete one-loop effective QED Lagrangian)

$$
\begin{aligned}
& \mathcal{L}_{E-H}=\mathcal{L}_{c l}+\frac{2 m^{4}}{\pi^{2}}\left(\frac{e a}{m^{2}}\right)^{4} \\
& \times \sum_{n=0}^{\infty} \frac{2^{2 n} B_{2 n+4}}{(2 n+2)(2 n+3)(2 n+4)}\left(\frac{e a}{m^{2}}\right)^{2 n}
\end{aligned}
$$

where $m$ is the electron mass and $B_{n}$ is the Bernoulli number. Clearly the series (13) is an asymptotic series which is divergent, so that for ultra strong magnetic field problems one can not apply it for computational purposes. For instance, for the critical value $B_{c r}$ of the magnetic field, which supposed to be in the cores of some neutron stars, the series in the Euler-Heisenberg effective Lagrangian (13) gives alternative in sign values when we include more than three terms in the truncated series.

In this section we evaluate the light velocity using the series representation for the one-loop effective Lagrangian, Eq. (3), in weak and strong magnetic field regions and derive asymptotic formulae in the ultra strong field regime.

Without loss of generality we choose the magnetic field to be directed along the $z$-axis, so that $\vec{B}=(0,0, a)$. The non-vanishing components of the classical and quantum energy-momentum tensor are the following

$$
\begin{aligned}
T_{c l}^{00}= & T_{c l}^{11}=T_{c l}^{22}=-T_{c l}^{33}=\frac{a^{2}}{2} \\
<T^{00}>= & -<T^{33}>=\frac{a^{2}}{2}-\delta \mathcal{L}_{m}, \\
<T^{11}>= & <T^{22}>=-<T^{33}> \\
= & \frac{a^{2}}{2}-\delta \mathcal{L}_{m}-a \partial_{a} \delta \mathcal{L} .
\end{aligned}
$$

Hereafter in this section the functions $\partial_{a} \delta \mathcal{L}, \partial_{b} \delta \mathcal{L}, \partial_{a}^{2} \delta \mathcal{L}$, $\partial_{b}^{2} \delta \mathcal{L}$ are taken in the limit of vanishing electric field, $b \rightarrow 0$,

$$
\begin{aligned}
\partial_{a} \delta \mathcal{L} & =-\frac{e^{2} a}{2 \pi^{4}} G(a)-\frac{e^{2} a^{2}}{4 \pi^{4}} G^{\prime}(a) \\
\partial_{b} \delta \mathcal{L} & =0 \\
\partial_{a}^{2} \delta \mathcal{L} & =-\frac{e^{2}}{2 \pi^{4}} G(a)-\frac{e^{2} a}{\pi^{4}} G^{\prime}(a)-\frac{e^{2} a^{2}}{4 \pi^{4}} G^{\prime \prime}(a),
\end{aligned}
$$

$$
\begin{aligned}
\partial_{b}^{2} \delta \mathcal{L}= & \frac{e^{4} a^{2}}{36 \pi^{2} m^{4}}+\frac{e^{4} a^{3}}{3 \pi^{4} m^{4}} G^{\prime}(a) \\
& +\frac{e^{4} a^{4}}{6 \pi^{4} m^{4}} G^{\prime \prime}(a)
\end{aligned}
$$

where

$$
G(a)==\sum_{n=1}^{\infty} \frac{1}{n^{2}} g\left(\frac{n \pi m^{2}}{e a}\right) .
$$

The function $G(a)$ defines the one-loop contribution to the effective Lagrangian with a pure magnetic background and can be written in terms of the generalized Hurvitz $\zeta$-function. From Eq. (4) one can find immediately that the velocity of light propagating along the magnetic field remains unchanged [9, 11] whereas for the photon moving in the plane orthogonal to the magnetic field one has the phase velocity given by

$$
\bar{v}^{2}=\frac{1-Q\left(<T^{00}>+\frac{1}{2}<T_{\mu}^{\mu}>\right)}{1+Q<T^{00}>} .
$$

The VEVs of the energy-momentum tensor can be written in terms of the main function $G(a)$

$$
\begin{aligned}
<T^{00}> & =\frac{a^{2}}{2}-\delta \mathcal{L}_{m}, \\
<T_{\mu}^{\mu}> & =\frac{e^{2} a^{3}}{2 \pi^{4}} G^{\prime}(a), \\
\delta \mathcal{L}_{m} & =-\frac{e^{2} a^{2}}{4 \pi^{4}} G(a) .
\end{aligned}
$$

It should be noted that after taking the limit $b \rightarrow 0$ in the general formulae (9) we have obtained a non-trivial contribution from the non-vanishing term $\left.\partial_{b}^{2} \mathcal{L}\right|_{b \rightarrow 0}$. This contribution provides the leading decrease factor in the dependency of the light velocity in the strong field region. After simplification the final expression for the light velocity takes the form

$$
\begin{aligned}
\bar{v}^{2}= & \frac{2 a-\partial_{a} \delta \mathcal{L}-a \partial_{a}^{2} \delta \mathcal{L}}{2 a-\partial_{a} \delta \mathcal{L}+a \partial_{b}^{2} \delta \mathcal{L}} \\
= & \left(2+\frac{e^{2}}{\pi^{4}} G(a)+\frac{5 e^{2} a}{4 \pi^{4}} G^{\prime}(a)+\frac{e^{2} a^{2}}{4 \pi^{4}} G^{\prime \prime}(a)\right) \\
& \left(2+\frac{e^{4} a^{2}}{36 \pi^{2} m^{4}}+\frac{e^{2}}{2 \pi^{4}} G(a)+\left(\frac{e^{2} a}{4 \pi^{4}}+\frac{e^{4} a^{3}}{3 \pi^{4} m^{4}}\right) G^{\prime}(a)\right. \\
& \left.+\frac{e^{4} a^{4}}{6 \pi^{4} m^{4}} G^{\prime \prime}(a)\right)^{-1} .
\end{aligned}
$$

To compare our results with the results obtained in past we consider the light velocity in weak field approximation which can be obtained using the truncated one-loop effective Euler-Heisenberg Lagrangian [2, 11]

$$
\begin{aligned}
& \tilde{\mathcal{L}}_{E-H}=\mathcal{L}_{c l}+\frac{\tilde{c}_{1}}{4}\left(b^{2}-a^{2}\right)^{2}+\tilde{c}_{2} a^{2} b^{2}, \\
& \tilde{c}_{1}=\frac{8 \alpha_{0}^{2}}{45 m^{2}}, \\
& \tilde{c}_{2}=\frac{14 \alpha_{0}^{2}}{45 m^{2}} .
\end{aligned}
$$




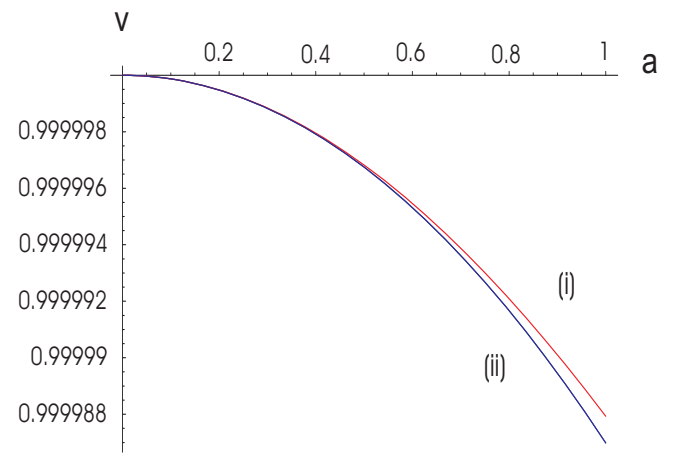

FIG. 1: Light velocity in the plane orthogonal to the magnetic field: (i) exact one-loop approximated result; (ii) result in the weak field approximation. The magnetic field strength $a$ is measured in units of electron mass $\mathrm{m}^{2}$.

For the photon propagating in the plane orthogonal to the magnetic field one can derive as in [11] the following expression for the velocity

$$
\bar{v}=1-\frac{11 \alpha_{0}^{2}}{45 m^{4}} B^{2}
$$

here $\alpha_{0}=\frac{e^{2}}{4 \pi \epsilon_{0}}=1 / 137.036$ is a fine structure constant. The corresponding light velocities obtained from the exact one-loop effective Lagrangian (3) and one-loop Euler-Heisenberg Lagrangian (20) are plotted in Fig. 1.

Now, let us consider light velocity in the strong field region. From the asymptotic behaviour of the main special functions $\operatorname{ci}(x), \operatorname{si}(x)[13$, 14] one can find the asymptotic formula for the function $G(a)$

$$
\begin{aligned}
G(a)= & -\frac{\pi^{2}}{6}\left(\ln \frac{e a}{m^{2}}+c_{1}\right)-\frac{\pi^{2} m^{2}}{2 e a}\left(\ln \frac{e a}{\pi m^{2}}+1\right) \\
& -\frac{\pi^{2} m^{4}}{4 e^{2} a^{2}}\left(\ln \frac{2 e a}{\pi m^{2}}-\gamma+\frac{5}{2}\right), \\
c_{1}= & -\gamma-\ln \pi+\frac{6}{\pi^{2}} \zeta^{\prime}(2)=-2.29191 \ldots .
\end{aligned}
$$

With this one results in the following asymptotic equation for the light velocity in a strong magnetic field

$$
\begin{gathered}
\bar{v}^{2} \simeq \frac{1-\frac{e^{2}}{12 \pi^{2}}\left(\ln \frac{e a}{m^{2}}+c_{1}+1\right)}{1-\frac{e^{2}}{12 \pi^{2}}\left(\ln \frac{e a}{m^{2}}+c_{2}\right)+\frac{e^{3} a}{24 \pi^{2} m^{2}}}, \\
c_{2}=\ln 2 \pi+c_{1}+\frac{3}{2}-\gamma=-1.8207048 \ldots
\end{gathered}
$$

The last term in the denominator in Eq. (23) gives a linear decrease of light velocity ( for strong magnetic fields $\left.a>B_{c r}=\frac{m^{2}}{e}\right)$ in qualitative agreement with results in [9, 11].
Notice that the trace anomaly $<T^{\mu}{ }_{\mu}>$ of the energymomentum plays a crucial role in providing the upper bound for the factor $Q\left(<T^{00}>+\frac{1}{2}<T_{\mu}^{\mu}>\right)<1$ until $a$ reaches the value of the Landau pole. Our formula improves on previously known expressions for the light velocity [9, 11] and can be applied to ultra strong magnetic fields.

\section{LIGHT VELOCITY IN HOMOGENEOUS ELECTRIC BACKGROUND}

Let us consider an homogeneous electric field $\vec{E}=$ $(0,0, b)$ directed along the $z$-axis. The non-vanishing components of the energy-momentum tensor and its VEVs are

$$
\begin{gathered}
T^{00}=T^{11}=T^{22}=-T^{33}=\frac{b^{2}}{2}, \\
<T^{00}>=-<T^{33}>=\frac{b^{2}}{2}-\delta \mathcal{L}_{e l}+b \partial_{b} \delta \mathcal{L}, \\
<T^{11}>=<T^{22}>=\frac{b^{2}}{2}+\delta \mathcal{L}_{e l},
\end{gathered}
$$

As in the case of a pure magnetic background the velocity of light propagating along the electric field remains unchanged and in the directions orthogonal to the electric field we have for the light velocity the same equation (16). The formula for the light velocity derived from the one-loop Euler-Heisenberg effective Lagrangian coincides with one expressed by Eq. (19) with the replacement $B \rightarrow E$. The explicit expressions for the effective action charge $Q$ and VEVs of the components $\left\langle T^{00}\right\rangle,\left\langle T_{\mu}^{\mu}\right\rangle$ can be derived in a similar manner as in the previous section

$$
\begin{aligned}
Q & =\frac{\partial_{a}^{2} \mathcal{L}+\partial_{b}^{2} \mathcal{L}}{\mathcal{L}_{e l}\left(\partial_{a}^{2} \mathcal{L}+\partial_{b}^{2} \mathcal{L}\right)+\left(\partial_{b} \mathcal{L}\right)^{2}-b \partial_{b} \mathcal{L} \partial_{a}^{2} \mathcal{L}}, \\
\mathcal{L}_{e l} & =\frac{b^{2}}{2}+\frac{e^{2} b^{2}}{4 \pi^{4}} H(b), \\
\partial_{a} \delta \mathcal{L} & =0 \\
\partial_{b} \delta \mathcal{L} & =\frac{e^{2} b}{2 \pi^{4}} H(b)+\frac{e^{2} b^{2}}{4 \pi^{4}} H^{\prime}(b), \\
\partial_{a}^{2} \delta \mathcal{L} & =\frac{e^{4} b^{2}}{36 \pi^{2} m^{4}}+\frac{e^{4} b^{3}}{3 \pi^{4} m^{4}} H^{\prime}(b)+\frac{e^{4} b^{4}}{6 \pi^{4} m^{4}} H^{\prime \prime}(b), \\
\partial_{b}^{2} \delta \mathcal{L} & =\frac{e^{2}}{2 \pi^{4}} H(b)+\frac{e^{2} b}{\pi^{4}} H^{\prime}(b)+\frac{e^{2} b^{2}}{4 \pi^{4}} H^{\prime \prime}(b), \\
<T^{00}> & =\frac{b^{2}}{2}+\frac{e^{2} b^{2}}{4 \pi^{4}} H(b)+\frac{e^{2} b^{3}}{4 \pi^{4}} H^{\prime}(b), \\
<T^{\mu}{ }_{\mu}> & =-\frac{e^{2} b^{3}}{2 \pi^{4}} H^{\prime}(b) .
\end{aligned}
$$

The function $H(b)$ is defined by

$$
H(b)=\sum_{n=1}^{\infty} \frac{1}{n^{2}} h\left(\frac{n \pi m^{2}}{e b}\right) .
$$




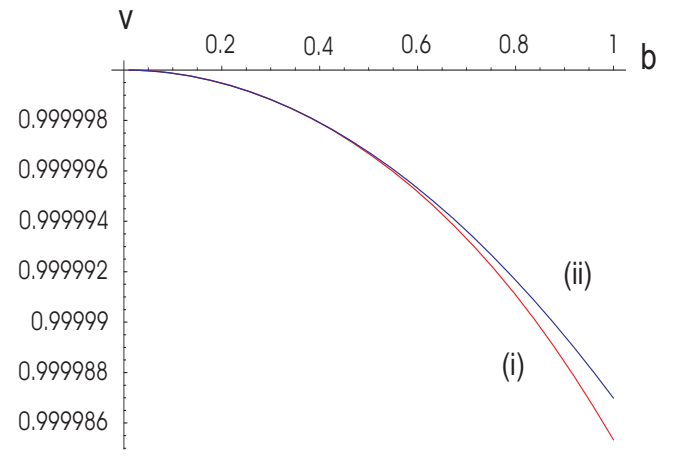

FIG. 2: Light velocity in the orthogonal plane to the electric field: (i) the exact one-loop approximated result; (ii) the result obtained in weak field approximation. The electric field strength $b$ is given in units of electron mass $m^{2}$.

After simplifications we find the following equation for light velocity in the orthogonal plane to the electric field

$$
\begin{aligned}
\bar{v}^{2}= & \frac{2 b+\partial_{b} \delta \mathcal{L}-b \partial_{a}^{2} \delta \mathcal{L}}{2 b+\partial_{b} \delta \mathcal{L}+b \partial_{b}^{2} \delta \mathcal{L}} \\
= & \left(2-\frac{e^{4} b^{2}}{36 \pi^{2} m^{4}}+\frac{e^{2}}{2 \pi^{4}} H(b)+\left(\frac{e^{2} b}{4 \pi^{4}}-\frac{e^{4} b^{3}}{3 \pi^{4} m^{4}}\right) H^{\prime}(b)\right. \\
& \left.-\frac{e^{4} b^{4}}{6 \pi^{4} m^{4}} H^{\prime}(b)\right)\left(2+\frac{e^{2}}{\pi^{4}} H(b)+\frac{5 e^{2} b}{4 \pi^{4}} H^{\prime}(b)\right. \\
& \left.+\frac{e^{2} b^{2}}{4 \pi^{4}} H^{\prime \prime}(b)\right)^{-1} .
\end{aligned}
$$

The exact values of the light velocity $\bar{v}$ in the weak field region $b \leq 1$ are plotted in Fig. 2 and can be compared with ones obtained from the one-loop Euler-Heisenberg Lagrangian (20).

Of course, for a strong electric field of magnitude above the critical value $E_{c r}=\frac{m^{2}}{e}$ pair creation, which we have not taken into account, becomes important, so in the strong field region one can only consider the light velocity dependence on the electric field $b$ formally. In that case the light velocity dependence is determined by the asymptotic behaviour of the function $H(b)$

$$
\begin{aligned}
H(b)= & -\frac{\pi^{2}}{6}\left(\ln \frac{e b}{m^{2}}+c_{1}\right)-\frac{\pi^{3} m^{2}}{4 e b}+ \\
& \frac{\pi^{2} m^{4}}{4 e^{2} b^{2}}\left(\ln \frac{2 e b}{\pi m^{2}}-\gamma+\frac{5}{2}\right),
\end{aligned}
$$

where we keep only leading terms.

For a strong electric field one has the following asymptotic formula

$$
\bar{v}^{2} \simeq \frac{1-\frac{e^{2}}{12 \pi^{2}}\left(\ln \frac{e b}{m^{2}}+c_{2}\right)}{1-\frac{e^{2}}{12 \pi^{2}}\left(\ln \frac{e b}{m^{2}}+c_{1}+1\right)} .
$$

In the asymptotic region approaching the Landau pole from the left the light velocity squared takes negative values. This shows that the Landau pole problem is still present.

\section{BIREFRINGENCE IN MAGNETIC FIELDS}

In this section we consider the birefringence effect with a complete one-loop effective Lagrangian (3). Let us choose the direction of the homogeneous magnetic field along the $z$-axis and the wave vector $\hat{\vec{k}}$ in the $x O z$-plane

$$
\begin{aligned}
\bar{k}^{\mu} & =(v, \sin \theta, 0, \cos \theta), \\
\epsilon_{\perp}^{\mu} & =(0,0,1,0), \\
\epsilon_{\|}^{\mu} & =(0,-\cos \theta, 0, \sin \theta),
\end{aligned}
$$

where $\theta$ is the angle between the vectors $\overrightarrow{\mathbf{B}}, \hat{\mathbf{k}}$ and $\epsilon_{\perp}^{\mu}, \epsilon_{\|}^{\mu}$ are polarization vectors in orthogonal and parallel directions to $\overrightarrow{\mathbf{B}}, \hat{\mathbf{k}}$-plane correspondingly.

To consider birefringence in the magnetic field we will start from the light cone condition Eq. (4). The non-zero components of the matrix $M_{\mu \nu}^{\alpha \beta}$ are

$$
\begin{aligned}
& M_{12}^{12}=a^{2} \partial_{x}^{2} \mathcal{L}, \\
& M_{03}^{12}=a^{2} \partial_{x y} \mathcal{L}, \\
& M_{03}^{03}=-a^{2} \partial_{y}^{2} \mathcal{L}, \\
& M_{12}^{03}=-a^{2} \partial_{x y} \mathcal{L} .
\end{aligned}
$$
follows

With this the light cone condition can be written as

$$
\begin{aligned}
\left(v^{2}-1\right) \partial_{x} \mathcal{L}= & a^{2} \partial_{y}^{2} \mathcal{L} v^{2} \epsilon_{3} \epsilon_{3}+M_{a b}^{m n} \hat{k}_{m} \hat{k}^{a} \epsilon_{n} \epsilon^{b}+ \\
& 2 M_{03}^{m n} v \hat{k}_{m} \epsilon_{n} \epsilon^{3} .
\end{aligned}
$$

For the $\perp, \|$-modes the last equation is simplified to

$$
\begin{aligned}
\left(v_{\perp}^{2}-1\right) \partial_{x} \mathcal{L} & =a^{2} \sin ^{2} \theta \partial_{x}^{2} \mathcal{L}, \\
\left(v_{\|}^{2}-1\right) \partial_{x} \mathcal{L} & =a^{2} \sin ^{2} \theta \partial_{y}^{2} \mathcal{L} v_{\|}^{2} .
\end{aligned}
$$

With this one can obtain the light velocities for $\perp$, $\|$ polarization modes

$$
\begin{aligned}
& v_{\perp}^{2}=1+\frac{a^{2} \sin ^{2} \theta \partial_{x}^{2} \mathcal{L}}{\partial_{x} \mathcal{L}}, \\
& v_{\|}^{2}=\frac{1}{1-\frac{a^{2} \sin ^{2} \theta \partial_{y}^{2} \mathcal{L}}{\partial_{x} \mathcal{L}}},
\end{aligned}
$$

or, equivalently,

$$
\begin{aligned}
& v_{\perp}^{2}=1+a \sin ^{2} \theta \frac{\partial_{a}^{2} \delta \mathcal{L}-\frac{1}{a} \partial_{a} \delta \mathcal{L}}{\partial_{a} \delta \mathcal{L}}, \\
& v_{\|}^{2}=\frac{1}{1+a \sin ^{2} \theta \frac{\partial_{b}^{2} \delta \mathcal{L}+\frac{1}{a} \partial_{a} \delta \mathcal{L}}{\partial_{a} \delta \mathcal{L}}} .
\end{aligned}
$$




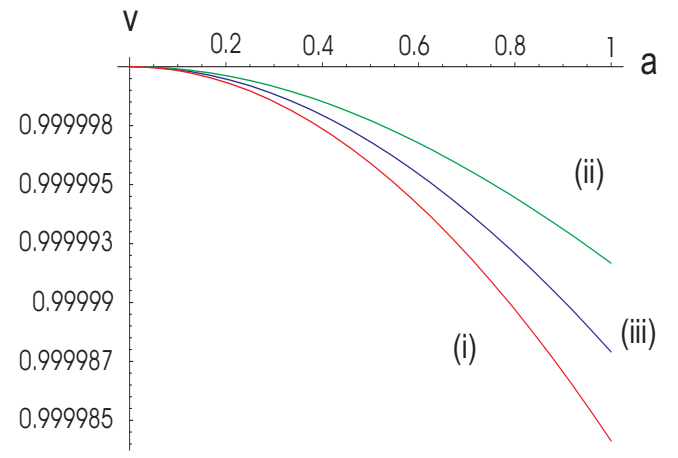

FIG. 3: The upper curve, (ii), corresponds to the $\|$-mode and the lower one, (i), corresponds to the $\perp$-mode. This can be compared with the light velocity averaged over polarizations, curve (iii).

For the special case $\theta=\frac{\pi}{2}$ the light velocities for $(\perp, \|)$ modes are plotted in Fig. 3 .

The estimated values for the velocities $v_{\perp, \|}$ can be compared with ones obtained in [9]. Tsai and Erber derived the explicit expressions for the refraction indices for $\perp, \|$ modes $(\theta=\pi / 2)$

$$
n_{\perp, \|}=1+\frac{\alpha}{4 \pi} \eta_{\perp, \|}(h)+o\left(\alpha^{2}\right),
$$

where $h=\frac{m^{2}}{2 e H}(H$ coincides with our notation $a)$ and $\eta$ functions are expressed in terms of special functions. This formula was derived approximately by neglecting terms of order higher than $\alpha$. So, the Tsai-Erber' formula is not valid for large magnetic fields $a>>1$. For weak fields our results are in a good quantative agreement with Tsai-Erber' results $\left(\eta_{T-E}\right)$ (see Table I).

\begin{tabular}{|c|l|l|c|r|}
\hline $\mathrm{h}$ & $\eta_{\|_{T-E}}$ & $\eta_{\| \text {our }}$ & $\eta_{\perp_{T-E}}$ & \multicolumn{1}{c|}{$\eta_{\perp \text { our }}$} \\
\hline 1 & 0.0705 & 0.07019 & 0.0335 & 0.03356 \\
\hline 2 & 0.0189 & 0.01879 & 0.0101 & 0.01014 \\
\hline 3 & 0.00852 & 0.008484 & 0.00472 & 0.004725 \\
\hline 4 & 0.00482 & 0.004801 & 0.00270 & 0.002708 \\
\hline 5 & 0.00309 & 0.003082 & 0.00175 & 0.001748 \\
\hline
\end{tabular}

TABLE I: Comparison of the Tsai and Erber results with our results for the functions $\eta_{\|, \perp}$.

For the strong field region $a>>1$ one can find the asymptotic formulae for the light velocities

$$
\begin{aligned}
& \bar{v}_{\perp}^{2} \simeq \frac{1-\frac{e^{2}}{12 \pi^{2}}\left(\ln \frac{e a}{m^{2}}+c_{1}+\frac{3}{2}\right)}{1-\frac{e^{2}}{12 \pi^{2}}\left(\ln \frac{e a}{m^{2}}+c_{1}+\frac{1}{2}\right)} \\
& \bar{v}_{\|}^{2} \simeq \frac{1-\frac{e^{2}}{12 \pi^{2}}\left(\ln \frac{e a}{m^{2}}+c_{1}+\frac{1}{2}\right)}{1-\frac{e^{2}}{12 \pi^{2}}\left(\ln \frac{2 e a}{\pi m^{2}}+1-\gamma\right)+\frac{e^{3} a}{12 \pi^{2} m^{2}}} .
\end{aligned}
$$

In the asymptotic limit $a \rightarrow \infty$ we have

$$
v_{\perp}^{2} \rightarrow 1, \quad \quad v_{\|}^{2} \rightarrow-\frac{m^{2}}{e a} \ln \left(\frac{e a}{m^{2}}\right) \rightarrow 0 .
$$

One can also find the corresponding refraction indices

$$
n_{\perp, \|}^{2}=\frac{1}{v_{\perp, \|}^{2}} .
$$

For instance, keeping only the leading linear term in (40) one can approximate the refraction index $n_{\|}$by

$$
n_{\|} \simeq \sqrt{1+\frac{e^{3} a}{12 \pi^{2} m^{2}}},
$$

in agreement with other authors 10, 15. Notice also that the birefringence effect was considered recently in 16] where the effective action approach [11] had been applied to a one-loop part of the Euler-Heisenberg effective Lagrangian (20).

\section{BIREFRINGENCE IN ELECTRIC FIELDS}

Let us choose the direction of the electric field along the $z$-axis, so that

$$
\begin{aligned}
& F^{\mu \nu}=0 \quad \text { except } F^{03}=|\overrightarrow{\mathbf{E}}| \equiv b, \\
& \tilde{F}^{\mu \nu}=0 \quad \text { except } \tilde{F}^{12}=b .
\end{aligned}
$$

The photon momentum $\hat{\mathbf{k}}$ and polarization vector $\epsilon$ are the same as ones considered for the pure magnetic case. The non-zero components of the matrix $M_{\mu \nu}^{\alpha \beta}$ are given by

$$
\begin{aligned}
& M_{12}^{12}=b^{2} \partial_{y}^{2} \mathcal{L}, \\
& M_{03}^{12}=-b^{2} \partial_{x y} \mathcal{L}, \\
& M_{03}^{03}=-b^{2} \partial_{x}^{2} \mathcal{L}, \\
& M_{12}^{03}=b^{2} \partial_{x y} \mathcal{L} .
\end{aligned}
$$

With this the light cone condition (4) can be rewritten as follows

$$
\begin{aligned}
\left(v^{2}-1\right) \partial_{x} \mathcal{L}= & b^{2} \partial_{x}^{2} \mathcal{L} v^{2} \epsilon_{3} \epsilon_{3}+M_{a b}^{m n} \hat{k}_{m} \hat{k}^{a} \epsilon_{n} \epsilon^{b} \\
& -2 M_{a b}^{03} v \hat{k}^{a} \epsilon^{b} \epsilon_{3} .
\end{aligned}
$$

For $\perp, \|$-modes the last equation gives

$$
\begin{aligned}
& \left(v_{\perp}^{2}-1\right) \partial_{x} \mathcal{L}=b^{2} \sin ^{2} \theta \partial_{y}^{2} \mathcal{L}, \\
& \left(v_{\|}^{2}-1\right) \partial_{x} \mathcal{L}=b^{2} \sin ^{2} \theta \partial_{x}^{2} \mathcal{L} v_{\|}^{2} .
\end{aligned}
$$

After changing variables $(x, y) \rightarrow(a, b)$ one obtains finally

$$
\begin{aligned}
& v_{\perp}^{2}=1-\sin ^{2} \theta\left(\frac{b \partial_{a}^{2} \delta \mathcal{L}}{\partial_{b} \delta \mathcal{L}}+1\right) \\
& v_{\|}^{2}=\frac{1}{1+\sin ^{2} \theta\left(\frac{b \partial_{b}^{2} \delta \mathcal{L}}{\partial_{b} \delta \mathcal{L}}-1\right)},
\end{aligned}
$$




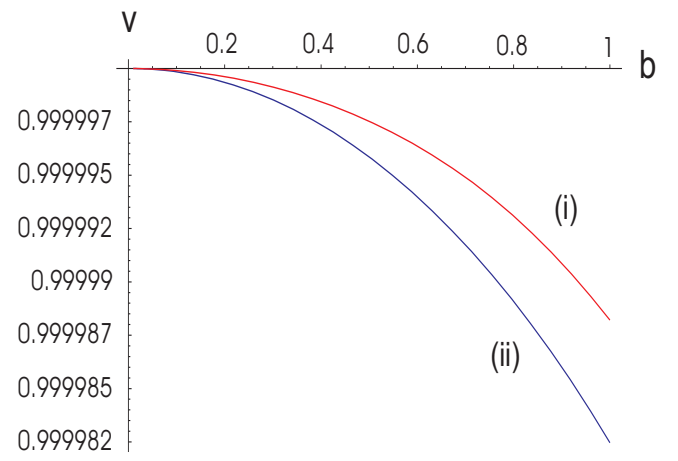

FIG. 4: Light velocity in the electric field for the $\|$-mode, (i), and for the $\perp$-mode, (ii).

where derivatives of the $\delta \mathcal{L}$ are taken at the limit $a=0$. For electric fields of magnitude below the critical value $b<1$ one has a small deviation of the light velocity for $\perp$, $\|$-modes from the velocity averaged over polarizations as it is pictured in Fig. 4.

\section{CONCLUSION}

In this paper we have demonstrated the applications of the analytic series representation for the one-loop effective action of QED proposed recently [5]. We have considered the light propagation effects in constant electric and magnetic fields and demonstrated the advantage of our effective action approach in comparison with the approach using the asymptotic series of Euler-Heisenberg effective Lagrangian (weak-field approximation of the complete one-loop effective Lagrangian) in the case of strong and ultra-strong magnetic fields. The analytical asymptotic formulae for light speed and the refractive indices for arbitrarily strong magnetic fields have been obtained. Even though our analysis is restricted to one-loop consideration, we hope that our results describe qualitatively the physical effects due to the very small value of the fine structure constant, and can provide good quantitative results in astrophysical problems concerning strong magnetic fields. The generalization to two-loop order is of further interest.

\section{APPENDIX}

\section{A. Notations and useful equations}

Our conventions for the metric tensor $g_{\mu \nu}$ and antisymmetric tensor $\epsilon^{\mu \nu \rho \sigma}$ are the following

$$
\begin{aligned}
g_{\mu \nu} & =(-+++), \\
\epsilon^{0123} & =-1, \\
\epsilon^{123} & =1 .
\end{aligned}
$$

We use Greek letters for 4-dimensional space-time coordinates and Latin letters for space indices only. One has useful identities

$$
\begin{aligned}
F_{\mu \rho} F_{\nu \sigma} F^{\rho \sigma} & =\frac{1}{2} F^{2} F_{\mu \nu}+\frac{1}{2}(F \tilde{F}) \tilde{F}_{\mu \nu} \\
F^{\mu \rho} F_{\rho}^{\nu} & -\tilde{F}^{\mu \rho} \tilde{F}_{\rho}^{\nu}=2 x g^{\mu \nu} \\
F^{\mu \rho} \tilde{F}_{\rho}^{\nu} & =\tilde{F}^{\mu \rho} F_{\rho}^{\nu}=y g^{\mu \nu} .
\end{aligned}
$$

Relations between the variables $a, b$ and $x, y$, and corresponding derivatives are given by

$$
\begin{aligned}
a= & \frac{1}{2} \sqrt{\sqrt{F^{4}+(F \tilde{F})^{2}}+F^{2}}=\sqrt{\sqrt{x^{2}+y^{2}}+x}, \\
b= & \frac{1}{2} \sqrt{\sqrt{F^{4}+(F \tilde{F})^{2}}-F^{2}}=\sqrt{\sqrt{x^{2}+y^{2}}-x,} \\
x= & \frac{1}{4} F_{\mu \nu} F^{\mu \nu}=\frac{1}{2}\left(a^{2}-b^{2}\right), \\
y= & \frac{1}{4} F_{\mu \nu} \tilde{F}^{\mu \nu}=a b, \\
\partial_{x}= & \frac{1}{\rho^{2}}\left(a \partial_{a}-b \partial_{b}\right), \\
\partial_{y}= & \frac{1}{\rho^{2}}\left(b \partial_{a}+a \partial_{b}\right), \\
\partial_{x}^{2}= & \frac{1}{\rho^{4}}\left(a^{2} \partial_{a}^{2}+b^{2} \partial_{b}^{2}-2 a b \partial_{a b}\right)+ \\
& \frac{1}{\rho^{6}}\left[\left(3 a b^{2}-a^{3}\right) \partial_{a}+\left(3 a^{2} b-b^{3}\right) \partial_{b}\right], \\
\partial_{y}^{2}= & \frac{1}{\rho^{4}}\left(b^{2} \partial_{a}^{2}+a^{2} \partial_{b}^{2}+2 a b \partial_{a b}\right)- \\
& \frac{1}{\rho^{6}}\left[\left(3 a b^{2}-a^{3}\right) \partial_{a}-\left(3 a^{2} b-b^{3}\right) \partial_{b}\right], \\
\partial_{x y}= & \frac{1}{\rho^{4}}\left(a b \partial_{a}^{2}-a b \partial_{b}^{2}+\left(a^{2}-b^{2}\right) \partial_{a b}\right)- \\
& \frac{1}{\rho^{6}}\left[\left(3 a^{2} b-b^{3}\right) \partial_{a}+\left(3 a b^{2}-a^{3}\right) \partial_{b}\right], \\
\rho^{2}= & a^{2}+b^{2} .
\end{aligned}
$$

Using the change of variables $(x, y) \rightarrow(a, b)$ one can express the matrix $M_{\alpha \beta}^{\mu \nu}$ in terms of variables $(a, b)$

$$
\begin{aligned}
& M_{\alpha \beta}^{\mu \nu}=\frac{1}{\rho^{4}}\left[a^{2} F^{\mu \nu} F_{\alpha \beta}+b^{2} \tilde{F}^{\mu \nu} \tilde{F}_{\alpha \beta}+a b\left(F^{\mu \nu} \tilde{F}_{\alpha \beta}\right.\right. \\
+ & \left.\left.\tilde{F}^{\mu \nu} F_{\alpha \beta}\right)\right] \partial_{a}^{2} \mathcal{L}+\frac{1}{\rho^{4}}\left[b^{2} F^{\mu \nu} F_{\alpha \beta}+a^{2} \tilde{F}^{\mu \nu} \tilde{F}_{\alpha \beta}\right. \\
- & \left.a b\left(F^{\mu \nu} \tilde{F}_{\alpha \beta}+\tilde{F}^{\mu \nu} F_{\alpha \beta}\right)\right] \partial_{b}^{2} \mathcal{L}+\frac{1}{\rho^{4}}\left[-2 a b F^{\mu \nu} F_{\alpha \beta}\right. \\
+ & \left.2 a b \tilde{F}^{\mu \nu} \tilde{F}_{\alpha \beta}+\left(a^{2}-b^{2}\right)\left(F^{\mu \nu} \tilde{F}_{\alpha \beta}+\tilde{F}^{\mu \nu} F_{\alpha \beta}\right)\right] \partial_{a b} \mathcal{L} \\
+ & \frac{1}{\rho^{6}}\left[\left(3 a b^{2}-a^{3}\right) F^{\mu \nu} F_{\alpha \beta}-\left(3 a b^{2}-a^{3}\right) \tilde{F}^{\mu \nu} \tilde{F}_{\alpha \beta}\right. \\
- & \left.\left(3 a^{2} b-b^{3}\right)\left(F^{\mu \nu} \tilde{F}_{\alpha \beta}+\tilde{F}^{\mu \nu} F_{\alpha \beta}\right)\right] \partial_{a} \mathcal{L} \\
+ & \frac{1}{\rho^{6}}\left[\left(3 a^{2} b-b^{3}\right) F^{\mu \nu} F_{\alpha \beta}-\left(3 a^{2} b-b^{3}\right) \tilde{F}^{\mu \nu} \tilde{F}_{\alpha \beta}\right. \\
+ & \left.\left(3 a b^{2}-a^{3}\right)\left(F^{\mu \nu} \tilde{F}_{\alpha \beta}+\tilde{F}^{\mu \nu} F_{\alpha \beta}\right)\right] \partial_{b} \mathcal{L} .
\end{aligned}
$$


For completeness we write down the expressions for the energy-momentum tensor $T_{u \nu}$ and effective charge $Q$ in terms of the $x, y$ variables 11 ]

$$
\begin{gathered}
T_{\nu}^{\mu}=F^{\mu \rho} F_{\nu \rho}-x \delta_{\nu}^{\mu}, \\
<T^{\mu \nu}>=\frac{2}{\sqrt{-g}} \frac{\delta S}{\delta g_{\mu \nu}} \\
S=\int d^{4} x \sqrt{-g} \mathcal{L}, \\
<T^{\mu \nu}>=-T^{\mu \nu} \partial_{x} \mathcal{L}+g^{\mu \nu}\left(\mathcal{L}-x \partial_{x} \mathcal{L}-y \partial_{y} \mathcal{L}\right), \\
Q=\frac{1}{2}\left(\partial_{x}^{2} \mathcal{L}+\partial_{y}^{2} \mathcal{L}\right)\left(\left(\partial_{x} \mathcal{L}\right)^{2}+\left(\partial_{x} \mathcal{L}\right)\left(\frac{x}{2}\left(\partial_{x}^{2}-\partial_{y}^{2}\right)\right.\right. \\
\left.\left.+y \partial_{x y}\right) \mathcal{L}+\frac{1}{2}\left(\partial_{x}^{2}+\partial_{y}^{2}\right) \mathcal{L}\left(1-x \partial_{x}-y \partial_{y}\right) \mathcal{L}\right)^{-1}
\end{gathered}
$$

\section{B. Asymptotic formulae}

The asymptotic properties of standard special functions are given in 14]. Keeping the leading terms in series expansions we obtain the leading terms in asymptotic expansion of the main special functions: in $x \rightarrow \infty$ region

$$
\begin{aligned}
e^{-x} \operatorname{Ei}(x) & \rightarrow \frac{1}{x} \\
e^{x} \operatorname{Ei}(-x) & \rightarrow-\frac{1}{x} \\
g(x) & \rightarrow-\frac{1}{x^{2}}+\frac{6}{x^{4}} \\
h(x) & \rightarrow \frac{1}{x^{2}}+\frac{6}{x^{4}}
\end{aligned}
$$

in $x \rightarrow 0$ region

$$
\begin{aligned}
\operatorname{Ei}(x) & \rightarrow \gamma+\ln x, \\
\operatorname{Ei}(-x) & \rightarrow \gamma+\ln x, \\
\operatorname{ci}(x) & \rightarrow \gamma+\ln x, \\
\operatorname{si}(x) & \rightarrow-\frac{\pi}{2}, \\
g(x) & \rightarrow \gamma+\ln x, \\
h(x) & \rightarrow \gamma+\ln x .
\end{aligned}
$$

The asymptotic formulae for the functions $G(a)$, Eq. (22), and $H(b)$, Eq. (28), can be derived in several ways, either from Schwinger's original integral formula for the one-loop effective Lagrangian using the generalized Hurwitz $\zeta$-function, or using the asymptotic formula for $g(x)$ and the series representation Eq. (3). Consider the integral representations for the main functions

$$
\begin{aligned}
& g(x)=-\int_{0}^{\infty} \frac{t e^{-x t}}{1+t^{2}} d t \\
& h(x)=\int_{0}^{\infty} \frac{t e^{-x t}}{1-t^{2}} d t
\end{aligned}
$$

Substituting these expressions in Eqs. (16 26) and performing the summation one finds the integral representations

$$
\begin{aligned}
& G(a)=-\int_{0}^{\infty} \frac{d t t}{1+t^{2}} \operatorname{Li}_{2}\left(e^{-\frac{\pi m^{2}}{e a} t}\right), \\
& H(b)=\int_{0}^{\infty} \frac{d t t}{1-t^{2}} \operatorname{Li}_{2}\left(e^{-\frac{\pi m^{2}}{e b} t}\right)
\end{aligned}
$$

from which the asymptotic formulae for the functions $G(a), H(b)$ can be easily derived. The values of the functions $G(a), H(b)$ obtained from the series representation and from the asymptotic formulae for large $a, b \gg 1$ can be viewed in Table II.

\begin{tabular}{|l|l|l|l|l|l|}
\hline $\mathrm{a}$ & $G_{\text {series }}$ & $G_{\text {asym }}$ & $\mathrm{b}$ & $H_{\text {series }}$ & $H_{\text {asym }}$ \\
\hline $10^{1}$ & -0.37815 & -0.316198 & $10^{1}$ & +0.122089 & +0.081690 \\
\hline $10^{2}$ & -2.38586 & -2.385448 & $10^{2}$ & -2.082565 & -2.082954 \\
\hline $10^{3}$ & -5.71865 & -5.718648 & $10^{3}$ & -5.653113 & -5.653116 \\
\hline $10^{4}$ & -9.42814 & -9.428142 & $10^{4}$ & -9.41787 & -9.417870 \\
\hline $10^{5}$ & -13.2046 & -13.204571 & $10^{5}$ & -13.20317 & -13.203169 \\
\hline $10^{6}$ & -16.99072 & -16.990717 & $10^{6}$ & -16.99054 & -16.9905396 \\
\hline
\end{tabular}

TABLE II: Comparison of the results obtained from series representation with the results derived from the asymptotic formulae

\section{Acknowledgments}

One of the authors (YMC) thanks Professor C. N. Yang for some illuminating discussions. The work is supported in part by the Korea Research Foundation and by the BK21 project of the Ministry of Education. Some of this work while one of the authors (MLW) was working at the Department of Physics and Applied Physics at Kyung Hee Unversity.
[1] W. Heisenberg and H. Euler, Z. Phys. 98, 714 (1936); V. Weisskopf, Kgl. Danske Vid. Sel. Mat. Fys. Medd. 14, 6 (1936).

[2] V. Ritus, JETP 42, 774 (1976); 46, 423 (1977); M. Reuter, M. Schmidt, and C. Schubert, Ann. Phys, 259, 313 (1997).

[3] A. Nikishov, JETP 30, 660 (1970); W. Dittrich, J. Phys. A9, 1171 (1976); S. Blau, M. Visser, and A. Wipf, Int. J.
Mod. Phys. A6, 5409 (1991); J. S. Heyl and L. Hernquist, Phys. Rev. D55, 2449 (1997); C. Beneventano and E. Santangelo, J. Math. Phys. 42, 3260 (2001).

[4] W. Dittrich, H. Gies, Probing The Quantum Vacuum, Springer Tracts in Modern Physics, Vol. 166; Springer, 2000.

[5] Y. M. Cho and D. G. Pak, Phys. Rev. Lett. 86, 1947 (2001); W. Mielniczuk, J. Phys., A15, 2905 (1982). 
[6] J. Schwinger, Phys. Rev. 82, 664 (1951).

[7] S. L. Adler, Ann. Phys. (N. Y.) 67, 599 (1971).

[8] Z. Bialynicka-Birula and I. Bialynucki-Birula, Phys. Rev. D2, 2341 (1970); E. Brezin and C. Itzykson, Phys. Rev. D 3, 618 (1971).

[9] W.-Y. Tsai and T. Erber, Phys. Rev. D 12, 1132 (1975); W.-Y. Tsai, Phys. Rev. D 10, 2699 (1974); V. N. Baier, V. M. Katkov and V. M. Strakhovenko, ZhETF 68, 403 (1975).

[10] I. A. Batalin and A. E. Shabad, ZhETF 60, 894 (1971) (Sov. Phys. - JETP 33,483 (1971).

[11] W. Dittrich and H. Gies, Phys. Rev. D58, 025004 (1998); Phys. Lett. B 431, 420 (1998).
[12] V. M. Kaspi, Pulsar Astronomy - 2000 and Beyond. ASP Conference Series 202 (1999) (M. Kramer, N. Wex and R. Wielebinski, eds.), astro-ph/9912284

[13] I. S. Gradshtein and I. M. Ryzhik, Tables of integrals, Series and Products, Academic Press (1965).

[14] M. Abramowitz and I. A. Stegun, Handbook of Mathematical Functions, Dover Publ., Inc., NY (1972).

[15] A. E. Shabad, Interaction of Electromagnetic Radiation with Supercritical Magnetic Field, hep-th/0307214

[16] V. A. De Lorenci, R. Klippert, M. Novello and J. M. Salim, Pjys. Lett. B 482, 134 (2000). 